\title{
BMJ Open Types of working-life sequences among people recently diagnosed with multiple sclerosis in Sweden: a nationwide register-based cohort study
}

\author{
Chantelle Murley (D) , ${ }^{1}$ Petter Tinghög, ${ }^{1,2}$ Korinna Karampampa, ${ }^{1}$ Jan Hillert, ${ }^{3}$ \\ Kristina Alexanderson, ${ }^{1}$ Emilie Friberg ${ }^{1}$
}

To cite: Murley C, Tinghög P, Karampampa K, et al. Types of working-life sequences among people recently diagnosed with multiple sclerosis in Sweden a nationwide register-based cohort study. BMJ Open 2020;10:e039228. doi:10.1136/ bmjopen-2020-039228

- Prepublication history and additional material for this paper is available online. To view these files, please visit the journal online (http://dx.doi.org/10. 1136/bmjopen-2020-039228).

Received 08 April 2020 Revised 20 0ctober 2020 Accepted 20 November 2020

Check for updates

(c) Author(s) (or their employer(s)) 2020. Re-use permitted under CC BY-NC. No commercial re-use. See rights and permissions. Published by BMJ.

${ }^{1}$ Division of Insurance Medicine, Department of Clinical Neuroscience, Karolinska Institutet, 171-77 Stockholm, Sweden

${ }^{2}$ Department of Health Sciences, Swedish Red Cross University College, 141-21 Huddinge, Sweden

${ }^{3}$ Division of Neurology, Department of Clinical Neuroscience, Karolinska Institutet, Stockholm, 171-77 Sweden

Correspondence to Ms Chantelle Murley; chantelle.murley@ki.se

\section{ABSTRACT}

Objectives To explore sequences of annual states of activity and sickness absence (SA) or disability pension (DP) (SA/DP) among working-aged people with multiple sclerosis (PwMS) as well as characteristics associated with the identified types of working-life sequences. Design Nationwide Swedish register-based cohort study from 1 year prior to 5 years after the year of multiple sclerosis (MS) diagnosis.

Setting Sweden.

Participants PwMS diagnosed in 2008-2011 when aged 20-55 ( $n=2652,69.9 \%$ women).

Primary and secondary outcome measures Individuallevel sequences spanning 7 years were constructed with annual states regarding activity (income from paid work, student allowances, parental leave or unemployment compensation) and/or SA/DP. Types of working-life sequences were identified among the individuals' sequences using hierarchical cluster analysis with optimal matching dissimilarity measures.

Results Six types of working-life sequences were identified. The largest cluster, Stable High Activity, represented $48.4 \%$ of the cohort. Other types were: Stable High SA/DP (14.5\%); Other (4.5\%); and three types with mixed activity and varying SA/DP regarding the number of days/year and timing (32.6\%). Characteristics of the different identified types of sequences were subsequently investigated. All types of sequences had lower odds for university education (OR range: 0.18-0.72) compared with Stable High Activity. Increasingly higher odds of having anxiety/depression compared with Stable High Activity were observed across the types of sequences, by increasing proportions of SA/DP. Stable High SA/DP sequences were less likely than Stable High Activity to be prescribed MS drugs in the MS diagnosis year (OR $0.61 ; 95 \% \mathrm{Cl} 0.47$ to 0.78 ). All types of sequences had higher disposable income in the final study year than the first, except for Stable High SA/DP sequences (Swedish Krona 4669, 95\% Cl -1892 to 11230 ).

Conclusions Diversity in working life was influenced by sociodemographic and clinical characteristics resulting in different activity and SA/DP patterns across the six identified types of working-life sequences.
Strengths and limitations of this study

- The use of real-world microdata from high-quality nationwide administrative registers with individuallevel linkages ensured that the results are not influenced by recall bias and reduced selection bias.

- Sequence analysis is capable of capturing the complexity and dynamic nature of sickness absence and labour market participation in longitudinal studies by including multiple outcomes simultaneously over time.

- Sequence analysis is an exploratory method rather than a hypothesis testing method and accordingly one cannot adjust for potential confounders when identifying the types of sequences within the cohort.

- The study had relatively short observation time (7 years in total); however, this was a tradeoff with studying a recently diagnosed cohort of people with multiple sclerosis with the current social security regulations and treatment protocols.

\section{INTRODUCTION}

Multiple sclerosis (MS) is a disease of the central nervous system often involving both cognitive disability and mobility impairment. ${ }^{2}$ People with MS (PwMS) are to a larger extent than people without MS not in paid work or working with reduced hours, even at low levels of disability. ${ }^{13}$ Pfleger et al operationalised the term 'working life' to broadly capture the complex situation among working-aged PwMS, including participating in paid work, receiving early retirement or unemployment compensation, and total income. $^{3}$

Changes in working life among PwMS, including reductions in earnings and increases of sickness absence (SA) and disability pension (DP), have received some prior attention. ${ }^{3-6}$ The consequences of these changes are not limited to the individual themselves, but include the family, workplace, insurer and society. ${ }^{78}$ 
However, aspects of working life have largely been considered as single outcomes or at single time points, rather than simultaneously to reflect the complexity of living with a chronic illness. Sequence analysis has been shown to be complimentary to traditional methods when studying working life. ${ }^{9-11}$ A sequence is an ordered list of states, and this method allows for the identification of reoccurring patterns across individuals' sequences considering the ordering, timing and duration of the component states. ${ }^{9} 1012$ Thus, details of the common patterns of working life, including transitions between outcomes of activity and SA/DP, among working-aged PwMS can be described simultaneously. By grouping individuals' sequences, types of working-life sequences can be identified to classify and describe the heterogeneous and complex experiences among PwMS.

We aimed to explore the sequences of activity states among PwMS of working age, then to identify types of working-life sequences from the individuals' sequences, and finally to investigate characteristics associated with the identified types of working-life sequences.

\section{METHODS}

An explorative Swedish cohort study was conducted with seven years of annual observation from one year prior $\left(\mathrm{Y}_{-1}\right)$ to five years after $\left(\mathrm{Y}_{+5}\right)$ the year of MS diagnosis $\left(\mathrm{Y}_{0}\right)$.

\section{Data}

Individual-level pseudonymised microdata were linked from six nationwide registers maintained by the following three Swedish agencies:

National Board of Health and Welfare

- National Patient Register (NPR) ${ }^{13}$ : dates for inpatient and specialised outpatient healthcare for MS.

- Cause of Death Register ${ }^{14}$ : year of death.

- Swedish Cancer Register: dates.

- Swedish Prescribed Drug Register $(\mathrm{SPDR})^{15}$ : dates and types of prescribed drugs dispensed at pharmacies by the Anatomical Therapeutic Chemical (ATC) Classification System.

Statistics Sweden

- Longitudinal Integration Database for Health Insurance and Labour Market Studies ${ }^{16}$ : working-life outcome variables, residence in Sweden, sociodemographic characteristics and annual incomes.

Karolinska University Hospital

- Swedish Multiple Sclerosis Registry (SMSReg) ${ }^{17}{ }^{18}:$ MS diagnosis dates.

\section{Study population}

Individuals aged $20-55$ on the 31 December the year prior to having their first ever MS code (International Classification of Diseases 10th Revision (ICD-10) code G35, with no prior G35 or ICD-8/9 code 340) recorded in the NPR as a main or side diagnosis for healthcare in 2008-2011 were identified $(\mathrm{n}=3142)$.
Exclusion criteria: to assume the MS code represented a new diagnosis, individuals in the SMSReg with an MS diagnosis date earlier than 365 days to the NPR date were excluded $(\mathrm{n}=199)$ and individuals not living in Sweden the two years prior to $Y_{0}$ were excluded $(n=46)$. Individuals without at least one additional MS code in the NPR or MS according to the SMSReg were excluded $(n=245)$ as unconfirmed MS. This resulted in $2652 \mathrm{PwMS}$.

Individuals were followed annually for seven years $\left(\mathrm{Y}_{-1}\right.$ to $\mathrm{Y}_{+5}$ ). Censoring occurred from the year of emigration $(n=27)$ or death $(n=30)$. In total, $2595(97.9 \%)$ PwMS had complete observation and were included in the main analyses.

\section{Swedish social security system}

The universal Swedish social security system covers individuals with reduced work capacity due to disease or injury through SA and DP payments. All individuals living in Sweden from the age of 16, who have income from work, parental leave or unemployment compensation, and reduced work capacity due to disease or injury are eligible for SA. SA replaces up to $80 \%$ of lost income from paid work. After the first waiting day without reimbursement, the employer pays for SA for 13 days, with this period not covered in register data. Thereafter, the Social Insurance Agency pays. For people registered as unemployed or on parental leave, SA is paid from day two with one waiting day and self-employed have varying numbers of waiting days. All individuals aged 19-64 can be granted DP replacing up to $64 \%$ of lost income from paid work if having long-term or permanently reduced work capacity due to disease or injury. Both SA and DP can be granted for part-time or full-time of usual work hours $(25 \%, 50 \%$, $75 \%$ or $100 \%$ ), with the possibility for simultaneous parttime SA and DP.

Unemployment compensation is provided to those registered with the Swedish Public Employment Service as actively seeking and immediately available for employment, replacing up to $80 \%$ of lost income due to unemployment. Individuals receiving unemployment compensation are assumed to have work capacity. ${ }^{5}$

\section{Activity states}

Individuals were assigned seven activity states, one for each study year. In order of assignment, the possible mutually exclusive states were: Censor, SA/DP, Mixed: SA/DP 180+ days, Mixed: SA/DP 30-180 days, Activity and Other.

The Censor state was assigned to individuals who had died or emigrated in that year and all remaining study years. Individuals with a Censor state were only included in the sensitivity analyses.

We combined net days of SA and DP as SA/DP; for example, one day of $50 \% \mathrm{SA}$ and one day of $50 \% \mathrm{DP}$ equated to one SA/DP net day. The SA/DP state was defined as $\geq 364$ net days of SA/DP in the calendar year, representing reduced working capacity for the entire year. Two states with mixed activity and some SA/DP 
were constructed based on the number of net SA/DP days during the calendar year: Mixed: SA/DP 180+ days described a year predominately on SA/DP along with some activity and Mixed: SA/DP 30-180 days for a year mostly in activity but with some SA/DP.

The Activity state was defined as having a combined annual income from the following four sources above $24 \%$ of the 'price base amount' (an annually set amount used for indexation) ${ }^{19}$ : income from paid work, student allowances, compensated parental leave and/or the active unemployment compensation. These four income sources all assume that the individual has work capacity, irrespective of whether the income is derived from paid work. This assumption is premised on the fact that the individuals would otherwise be eligible for SA/DP if work capacity were reduced due to disease or injury. The lowest annual income from activity to be assigned this state ranged between SEK9925 and SEK11 125 Swedish krona (SEK) $(\approx € 940-1050)$. In each year, $86 \%-93 \%$ of the total income from activity comprised income from paid work.

Lastly, the Other state constituted individuals who were alive and registered as living in Sweden but did not meet the definitions for the preceding states, for example, homemakers or unemployed individuals who were ineligible for unemployment compensation.

Three individuals had one missing state which was imputed from the adjacent states.

\section{Cohort characteristics}

All sociodemographic characteristics were measured on the 31 December $Y_{-1}$. These included: sex (women/men); age (groups); country of birth (Sweden, yes/no); type of living area (large cities, medium sized towns, small/ rural); geographical area of residence (north, south, east); educational level (some university/college, yes/ no); type of work (manager, office work, manual labour, unclassified work, not in paid work); and family composition (married/cohabiting (yes/no), and living with children $($ age $<18)($ yes $/$ no) $)$.

Annual incomes are reported in 2015 values in SEK $(€ 1$ $\approx$ SEK9.3535) with adjustment for inflation. ${ }^{20}{ }^{21}$ Three incomes were reported for $\mathrm{Y}_{-1}$ and $\mathrm{Y}_{+5}$ : income from activity (paid work, student allowances, compensated parental leave and unemployment compensation), income from paid work and household individualised disposable income (DI) ${ }^{22}$ In activity (yes/no) and incomes in $\mathrm{Y}_{-1}$ from unemployment compensation, compensated parental leave and student allowances (yes/ no) were included.

A modified Rx-Risk Comorbidity Index ${ }^{23} 24$ was constructed using ATC codes from the SPDR to measure comorbidity in $\mathrm{Y}_{0}$. First, individuals in the Swedish Cancer Register (yes) in $Y_{0}$ were included within the index under malignancies $(n=18)$ because most cancer treatments are not included in the SPDR. Second, MS drugs available in 2011 were excluded from the index and instead measured separately for $\mathrm{Y}_{0}$, MS drugs (yes/no). Comorbidity was then classified as $0,1-2,3-4, \geq 5$ comorbidity categories. ${ }^{23}$ Information from the index categories was used to indicate anxiety/depression (yes/no) and pain (yes/no) for $\mathrm{Y}_{0}$, as both are often self-reported by PwMS, even early in the disease course. ${ }^{1}$

\section{Patient and public involvement statement}

This explorative observational study using pseudonymised microdata was performed without patient or public involvement. Informed consent was not applicable and the PwMS included were not involved in the study process.

\section{Statistical analyses}

The study cohort was described by frequencies and percentages.

In the sequence analysis, the study population consisted of 2595 individuals and five potential states were included. Fifty-seven participants were excluded due to death or emigration. Individual-level sequences of the annual activity states were constructed from $Y_{-1}$ to $Y_{+5}$. A sequence was defined as an ordered list of states and successive identical states expressed on a time axis. ${ }^{9}{ }^{12} 25$ A sequence index plot was constructed visualising the sequences.

The sequences were then grouped to identify of the types of working-life sequences within the cohort and respective membership to each type. First, optimal matching algorithms computed dissimilarity measures between the sequences, defined as the cost of the required operations to transform one sequence into another when they are aligned. ${ }^{125-28}$ Then, similar sequences were grouped, using hierarchical agglomerative cluster analysis with Ward's linkage algorithm and the dissimilarity measures. 252829

For the dissimilarity measures, a cost of 1 for insertion and deletion of states and data driven substitution costs derived from the observed state transition matrix (table 1) were applied. ${ }^{28}{ }^{29}$ Higher costs were assigned to substituting between states with rare transitions and lower costs for frequently occurring transitions. ${ }^{2628}$

We evaluated 2-12 clusters with commonly used measures of partition quality (see online supplemental file 1). ${ }^{30}$ Additionally, we looked at the hierarchical cluster tree diagram of resulting cluster solutions and membership sizes for the interest in including additional clusters as types of working-life sequences.

After assigning each individual to the relevant cluster, representing a type of working-life sequence, further analyses were conducted. The membership characteristics of the six identified types of working-life sequences were described by frequencies and percentages. The number of unique sequences, mean duration within each state and mean number of transitions between states were calculated in total, and by type of sequence. A sequence index plot was constructed for each type of working-life sequence to visualise the individual sequences included.

Multinomial logistic regression models were used to analyse the associations between the types of working-life sequences and membership characteristics with the Stable High Activity type as the reference. Results are reported 
Table 1 Observed activity state transition matrix* of the people with multiple sclerosis $(n=2595)$ with five activity states 1

\begin{tabular}{|c|c|c|c|c|c|}
\hline & SA/DP & Mixed: SA/DP $180+$ days & Mixed: SA/DP $30-180$ days & Activity & Other \\
\hline SA/DP to & 0.90 & 0.09 & 0.02 & 0.00 & 0.00 \\
\hline Mixed: SA/DP $180+$ days to & 0.22 & 0.58 & 0.15 & 0.05 & 0.01 \\
\hline Activity to & 0.00 & 0.03 & 0.11 & 0.85 & 0.01 \\
\hline Other to & 0.02 & 0.06 & 0.06 & 0.23 & 0.63 \\
\hline
\end{tabular}

*Each row sums to 1, with some rounding. This state transition matrix contains the observed transition rates pooled across the sequences; state transition probabilities may differ if looking at a specific position in the sequence due to the importance of timing within the sequence. †SA/DP: $\geq 364$ net days of sickness absence (SA)/disability pension (DP); Mixed: SA/DP 180+ days: predominately on SA/DP (>180 net days) but also some activity; Mixed: SA/DP 30-180 days: predominately in activity but with some SA/DP (30-180 net days); Activity: income from activity is defined as the combined sum of income from paid work, student allowances, unemployment compensation and parental leave, above $24 \%$ of the 'price base amount' (a set amount used for indexation in Sweden that changes with inflation each year); and Other: alive and living in Sweden but did not meet the definitions for the preceding states.

as crude and adjusted ORs with 95\% CI. Adjustment was for sex, age and the year of MS diagnosis.

Dependent t-tests estimated the mean differences with $95 \%$ CI of annual incomes in $\mathrm{Y}_{+5}$ with those in $\mathrm{Y}_{-1}$, in total and by type of sequence.

Sensitivity analyses were conducted with six activity states (Censor included) and 2652 PwMS to investigate the possible impact of death or emigration on the results.

Data management and analyses were performed in SAS V.9.4 except for sequence analyses, which were performed with R V.3.5.2 (TraMineR ${ }^{27}$ and WeightedCluster ${ }^{30}$ ).

\section{RESULTS}

Cohort characteristics are presented in table 2.

Among the 2595 individuals included in the main analyses, there were 633 unique sequences (figure 1). The three most frequently occurring sequences accounted for $43.7 \%$ of PwMS: $33.5 \%$ of individuals had the Activity state throughout; $5.6 \%$ had the SA/DP state throughout; and $4.6 \%$ had the Activity state throughout except for a Mixed: SA/DP 30-180 days state in $\mathrm{Y}_{0}$.

State timing was important. The entropy index increased from 0.54 to 0.79 , indicating that individuals were more likely to transition between states with time (see online supplemental file 2). Figure 1 visualises this increase in transitions. The Activity state was the most frequent state among PwMS for all study years, with a mean duration of 4.20 years, but annual proportions decreased across the study (see online supplemental file 2). SA/DP and the two mixed activity and SA/DP states increased in frequency, while the Other state was infrequent throughout. Individuals with Mixed: SA/DP 30-180 days were most likely to have the same state in the following year but were more likely to transition to Activity than Mixed: SA/DP $180+$ days (table 1 ).

Six types of working-life sequences were identified through cluster analysis of the individual sequences. Figure 2 presents the sequences included within each of the six identified types of working-life sequences and the number of individuals belonging to each type. The types describe different patterns of activity and extent of SA/DP in terms of days in a year and duration across study years, as well as sequence stability in terms of average number of transitions between states. The types are presented in order of being most represented with activity (Stable High Activity) to full-time SA/DP (Stable High SA/DP). The type of sequence with the largest membership was Stable High Activity $(48.4 \%, \mathrm{n}=1257)$, characterised as being in the Activity state throughout or with the Mixed: SA/DP 30-180 days state proximate to $\mathrm{Y}_{0}$. Unstable Medium Activity $(16.5 \%, \mathrm{n}=427)$ sequences had an increase in proportion of Mixed: SA/DP 30-180 days in $\mathrm{Y}_{0}$ with a continued mix of predominantly Activity and the states associated with mixed activity and varying levels of SA/DP. Unstable Low SA/DP $(7.2 \%, \mathrm{n}=188)$ sequences began with mostly Activity but from $\mathrm{Y}_{0}$ transitioned to be characterised by Mixed: SA/DP 30-180 days. Unstable medium SA/DP $(8.9 \%, \mathrm{n}=230)$ was characterised by increasing proportions of the Mixed: SA/DP $180+$ days state within the sequences and some Activity and Mixed: SA/DP 30-180 days earlier in the sequences. Stable High SA/DP $(14.5 \%, \mathrm{n}=377)$ was characterised by a high extent of SA/DP throughout and few state transitions. Lastly, the Other type $(4.5 \%, \mathrm{n}=116)$ sequences predominately comprised the Other state.

Stable High Activity, Stable High SA/DP and Other were more homogenous in terms of state representation (figure 2) and had fewer state transitions (means ranging 0.6-1.9) than the Unstable Medium Activity, Unstable Low SA/DP and Unstable Medium SA/DP types (2.5-2.6).

Table 2 includes the membership characteristics of each type of working-life sequence. The adjusted ORs and $95 \%$ CIs of membership characteristics of the types of sequences, with Stable High Activity as the reference group, are presented in table 3 (crude estimates in online supplemental file 3 ). Men had lower odds than women to be in Unstable Medium Activity, Unstable Low SA/ DP or Unstable Medium SA/DP compared with Stable High Activity (OR range 0.41-0.62). Individuals with some college/university level education had lower odds 


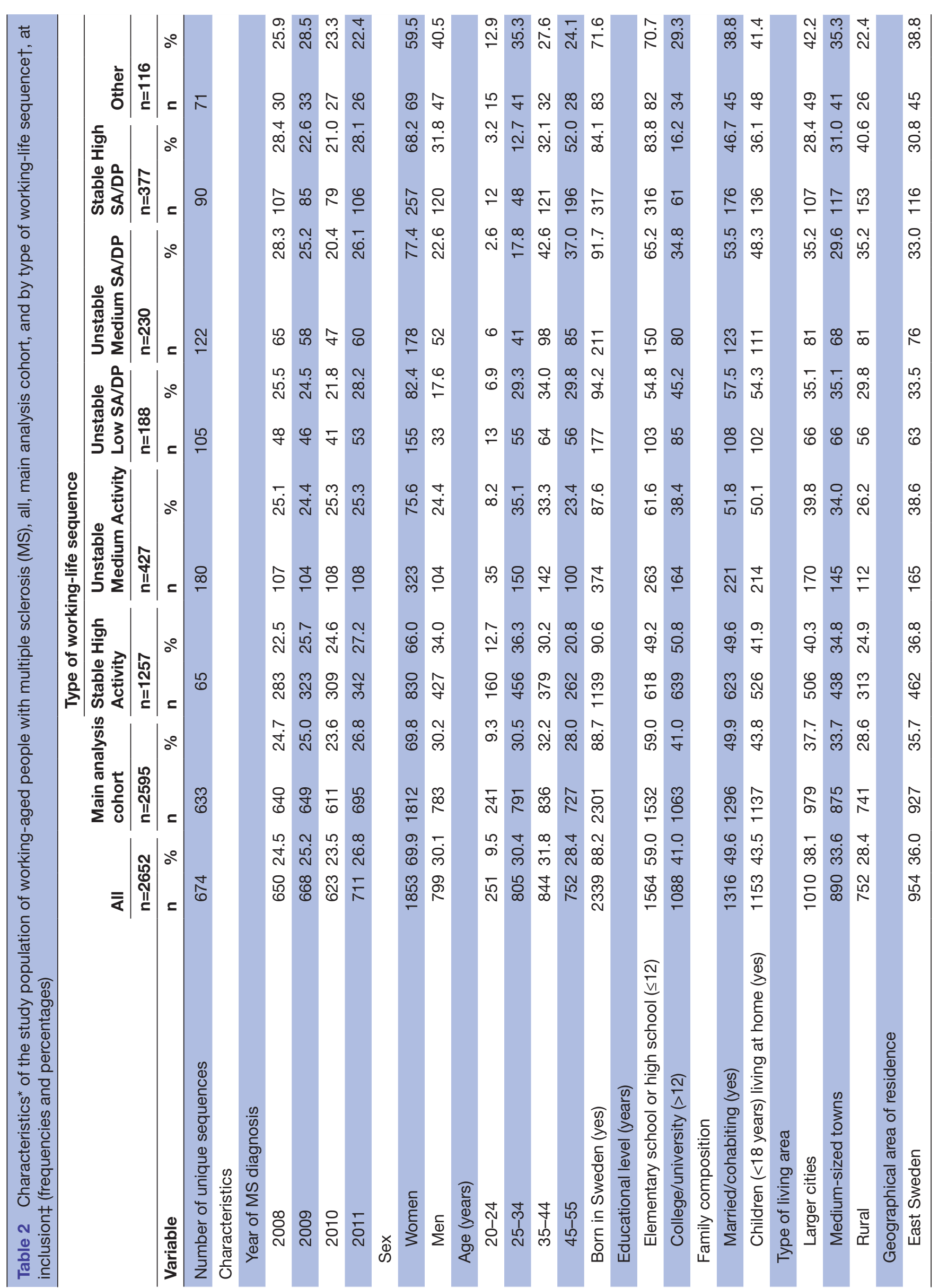




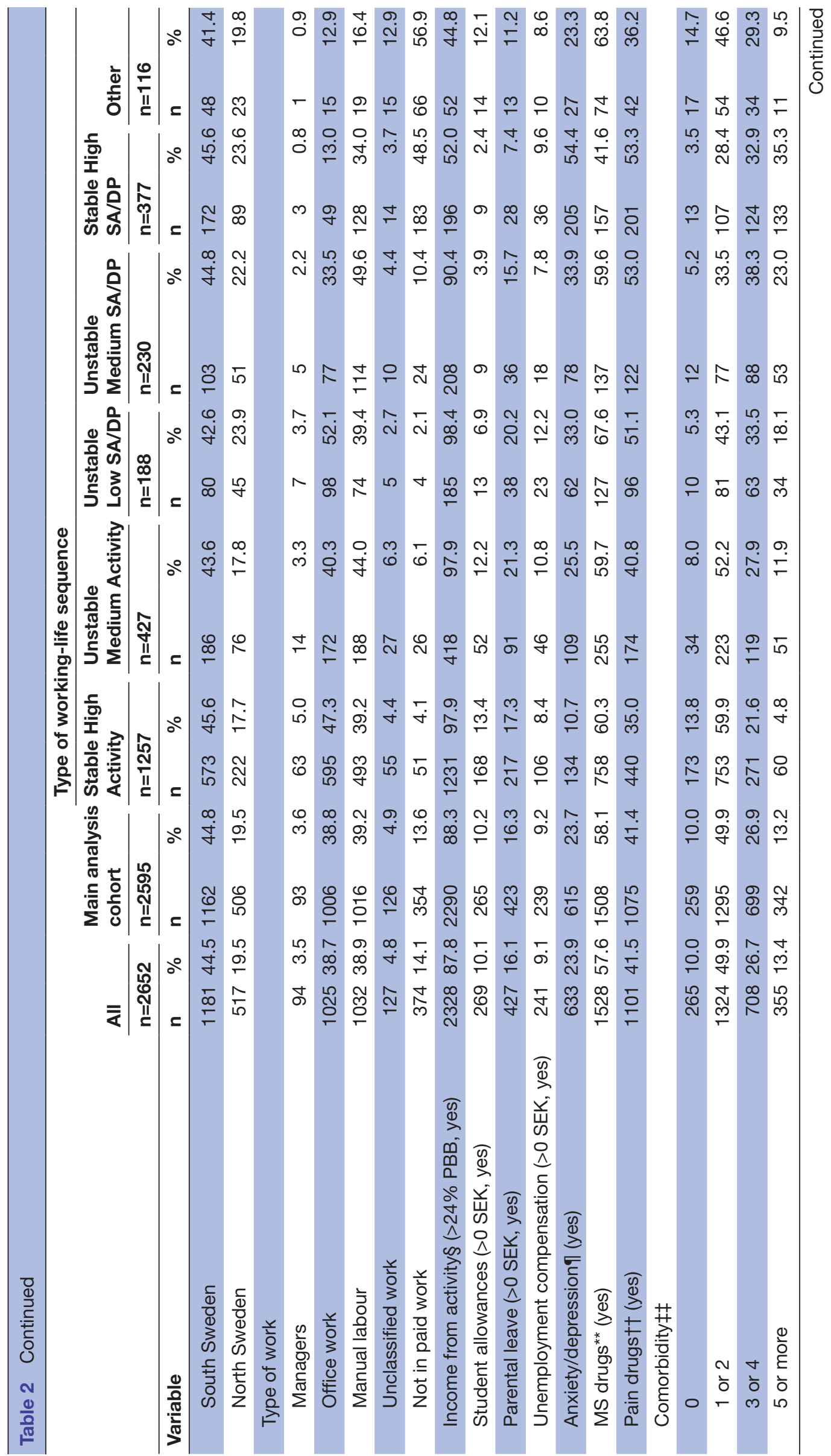


(OR range 0.18-0.72) than those with at most elementary school/high school to be in the types of sequences other than Stable High Activity. The odds of being in manual labour compared with office workers were higher for Unstable Medium Activity, Unstable Medium SA/ DP and Stable High SA/DP compared with Stable High Activity. Unstable Medium Activity to Stable High SA/DP had increasingly higher odds of having comorbidity and anxiety/depression compared with Stable High Activity. Those with Stable High SA/DP sequences were less likely than those with Stable High activity to be on MS medications in $\mathrm{Y}_{0}$ (OR $0.61 ; 95 \%$ CI 0.47 to 0.78 ), and those with Unstable Low SA/DP sequences more likely (OR 1.51; 95\% CI 1.08 to 2.11 ).

Overall, PwMS had increasing incomes from activity, paid work and DI comparing $Y_{+5}$ with $Y_{-1}$ (table 4). Stable High Activity sequences had a mean increase in annual income from activity comparing $\mathrm{Y}_{+5}$ with $\mathrm{Y}_{-1}$ (SEK105 911; $95 \%$ CI 99739 to 112 082). Unstable Low SA/DP, Unstable Medium SA/DP and Stable High SA/DP had less income from activity in $\mathrm{Y}_{+5}$ than in $\mathrm{Y}_{-1}$. Similar trends were observed regarding income from paid work. Among PwMS with income from activity in $Y_{+5}(n=2071), 93 \%$ of the income was from paid work. All types of working-life sequences had significantly higher mean DI in $\mathrm{Y}_{+5}$, except for Stable High SA/DP (SEK4669; 95\% CI -1892 to 11 230).

Sensitivity analyses of the entire PwMS cohort ( $\mathrm{n}=2652)$ with an additional state (Censor) suggest that the findings and interpretations would not have substantially differed (not presented). With six activity states, there were 674 unique sequences observed and the entropy index increased over the study period (from 0.5 to 0.75 ). The resulting six clusters were similar to the main analysis and cluster membership ranged from 117 to 1595 individuals. The largest difference was observed in cluster 5 (Unstable Medium Activity in the main analyses) with more heterogeneity in state representation.

\section{DISCUSSION}

In this Swedish register-based cohort study, working-life sequences focusing on how PwMS continues in activity with the flexible support of SA/DP were studied. Six types of working-life sequences were identified. Sociodemographic and clinical characteristics were associated with type of working-life sequence membership. These sequence types visualise the common patterns of work capacity among PwMS, including having work capacity throughout (ie, staying in activity), mixed sequences with activity and SA/DP proximate to MS diagnosis, increasing SA/DP over the study period or SA/DP of different extents throughout. Increasing diversity was observed across the sequences among those transitioning into states involving some extent of SA/DP, reflecting temporal changes in work capacity among PwMS and increasing heterogeneity along the clinical course. 


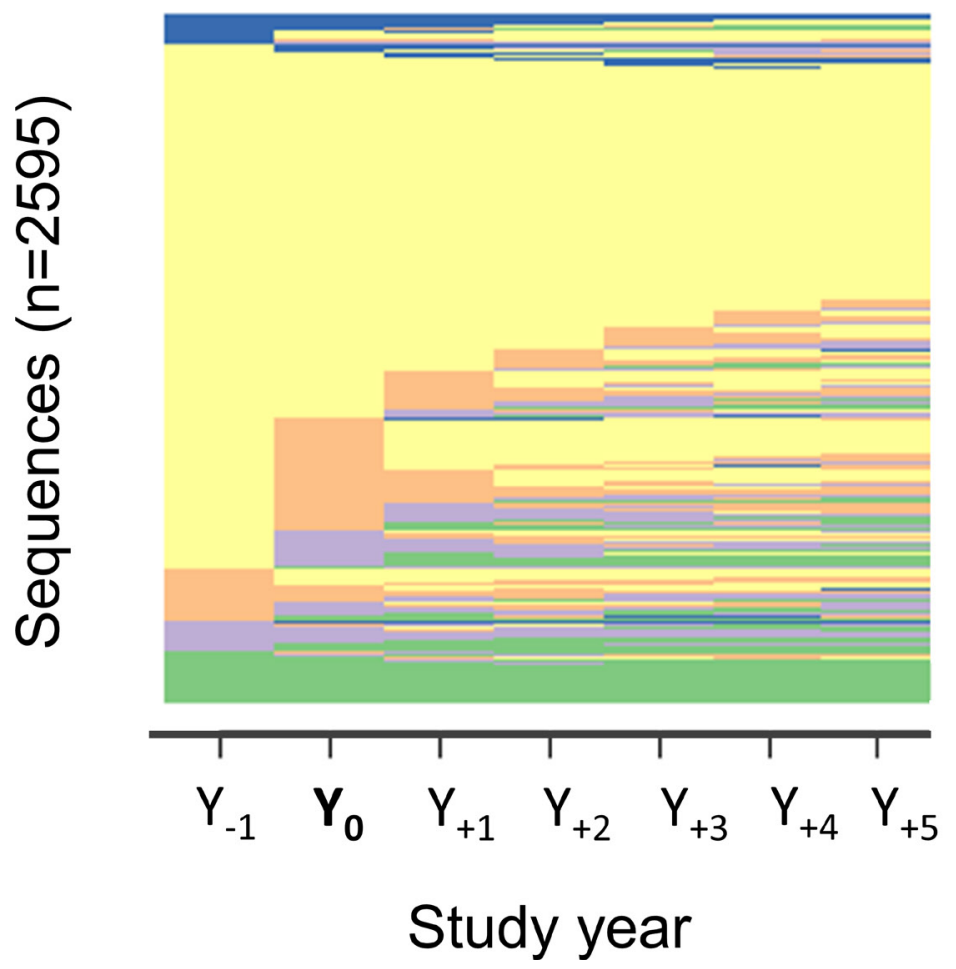

\begin{tabular}{|ll|}
\hline$\square$ & SA/DP \\
$\square$ & Mixed: SA/DP 180+ days \\
$\square$ & Mixed: SA/DP 30-180 days \\
$\square$ & Activity \\
$\square$ & Other \\
\hline
\end{tabular}

Figure 1 Sequence index plot of all the individuals' working-life sequences $(n=2595)$, sorted by the state assigned in $Y_{-1}$. DP, disability pension; SA, sickness absence.

The most frequent type of sequence was characterised by activity and was in accordance with previous findings that PwMS receive most of their income from paid work. ${ }^{5}$ Employment has previously been found to be associated with quality of life among PwMS, ${ }^{31}$ with PwMS in Stockholm reporting a preference in terms of quality of life to reduce hours, that is, mixed activity and
SA/DP, than to leave work completely. ${ }^{31}$ Despite the relative stability of Stable High Activity, some sequences in this cluster had mixed activity and SA/DP states proximate to MS diagnosis. Furthermore, shorter spells of SA $(<30$ days per calendar year paid by the Social Insurance Agency), were possible within the Activity state.
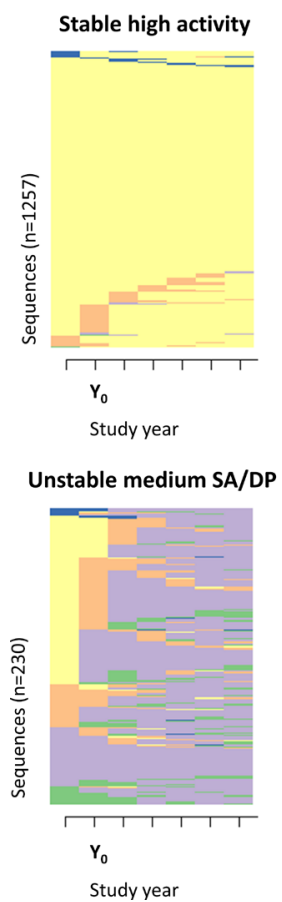
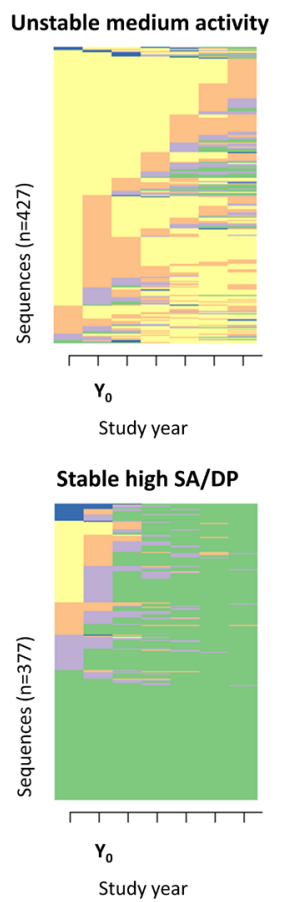
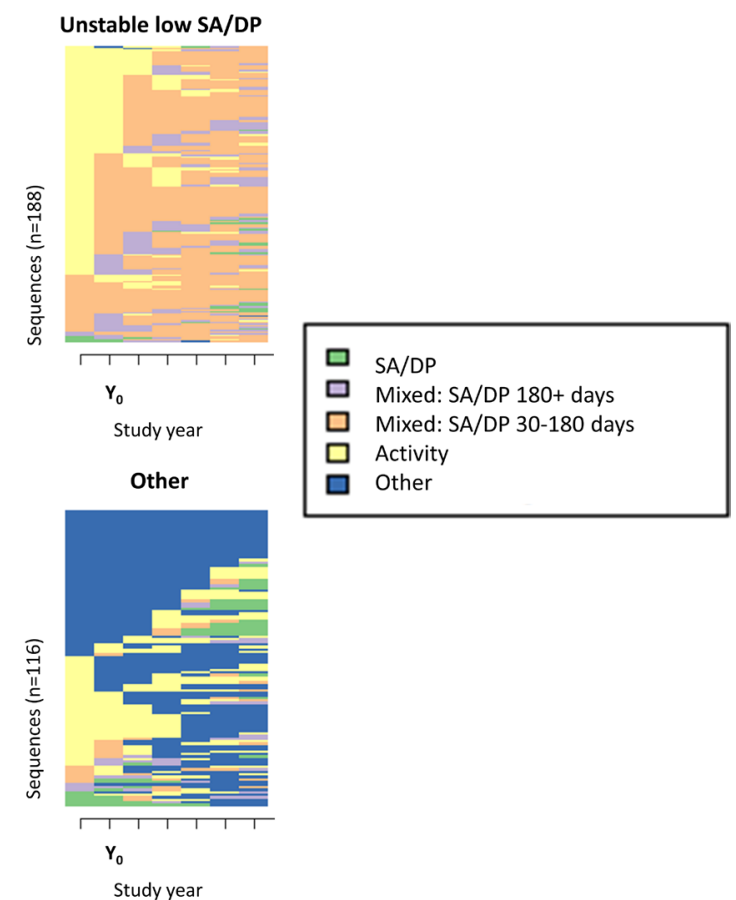

Figure 2 Sequence index plots of the six types of working-life sequences $(n=2595)$, sorted by the state assigned in $Y_{-1}$, and the number of individuals with each type of working-life sequence. DP, disability pension; SA, sickness absence. 


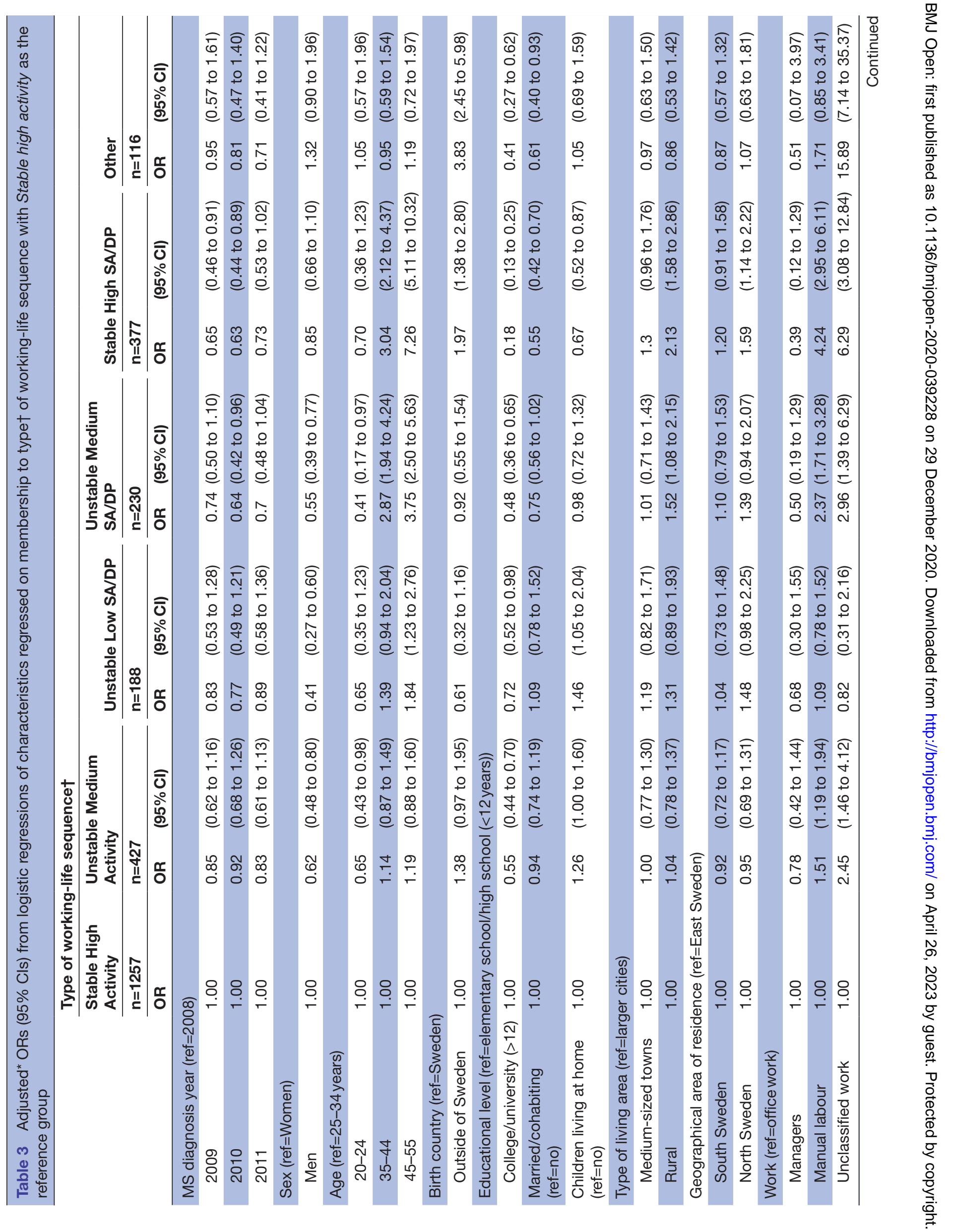




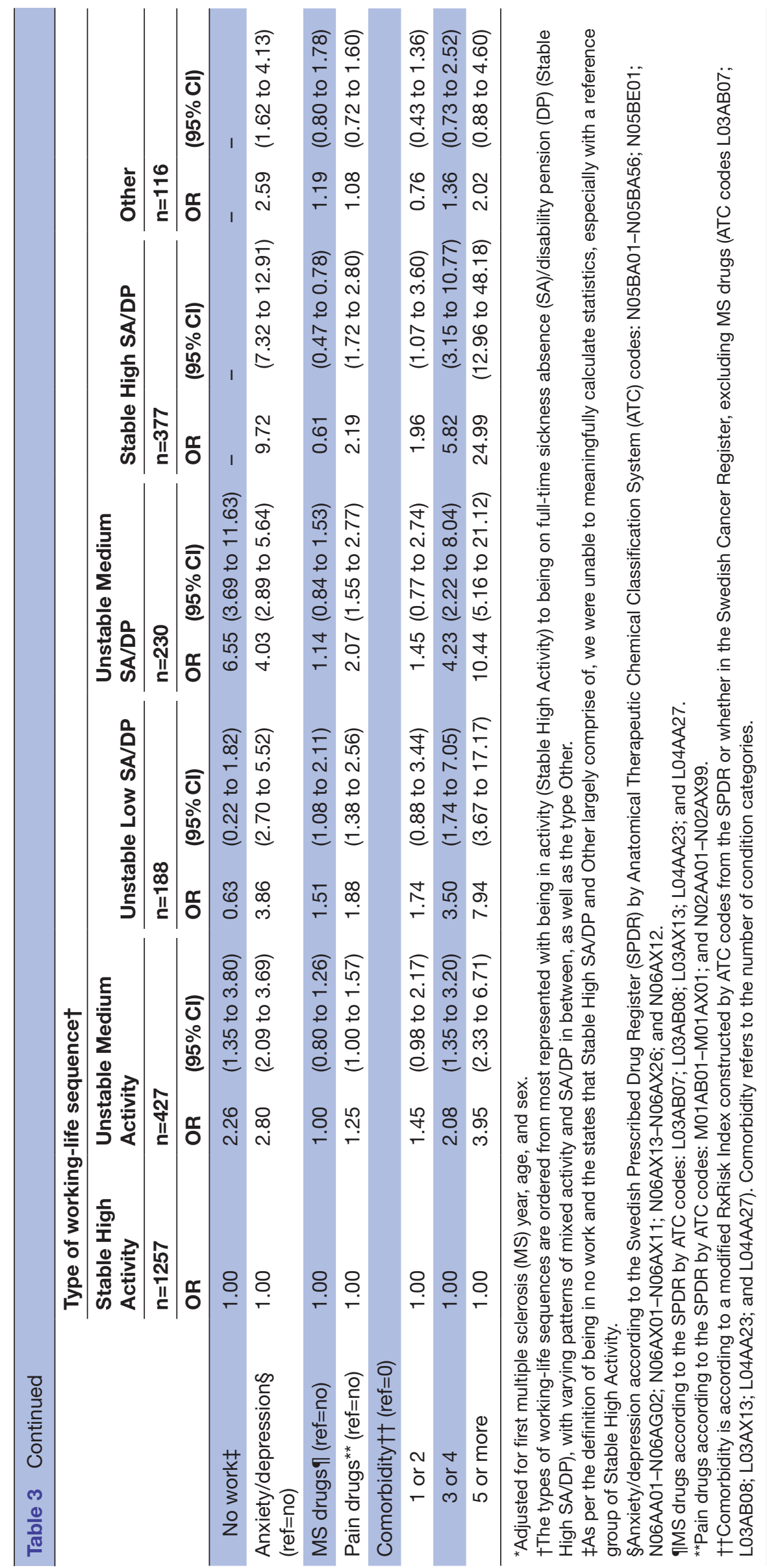




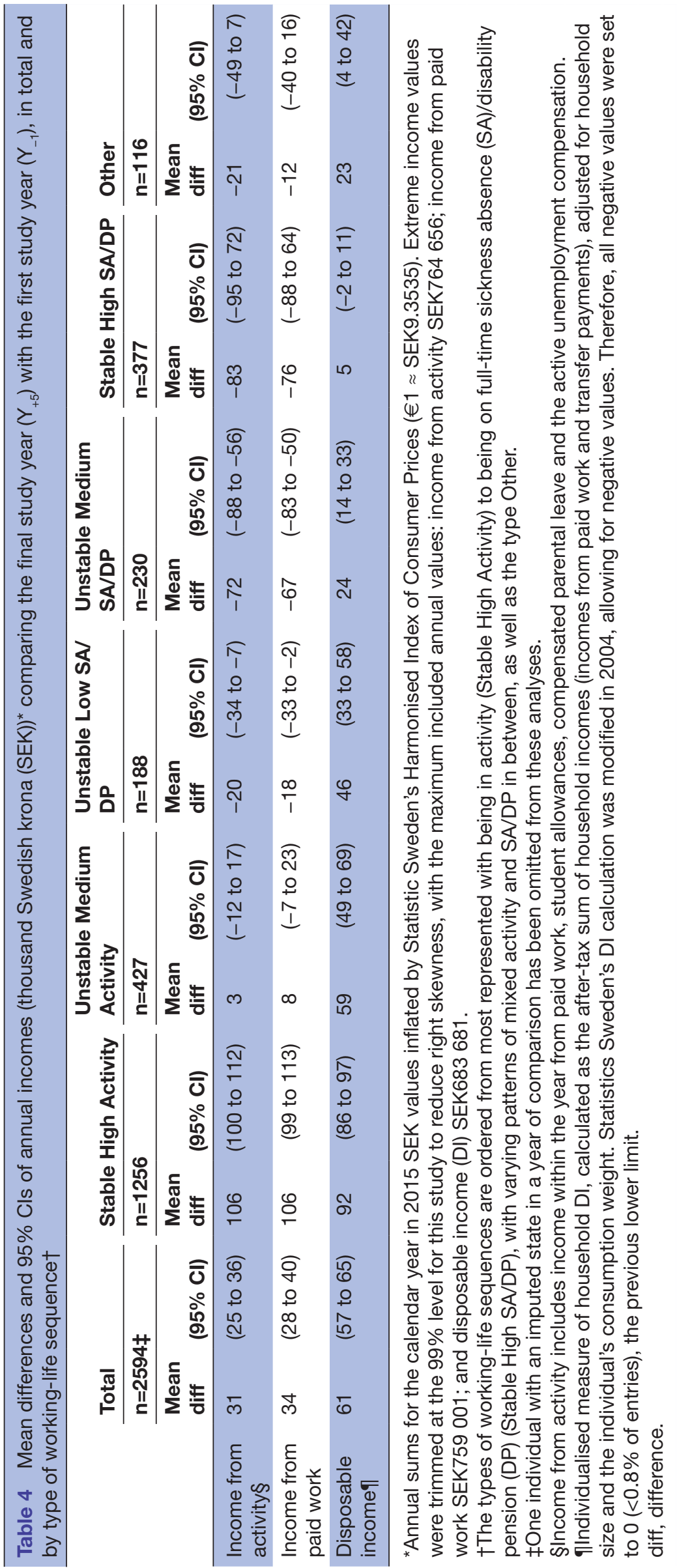


Unstable Medium Activity, Unstable Low SA/DP and Unstable Medium SA/DP were characterised by sequence instability and mixed activity and SA/DP. The extent of $\mathrm{SA} / \mathrm{DP}$ in terms of state timing within the sequence, duration and the number of annual SA/DP days differentiated these three types. These types of sequences were more heterogeneous and had more transitions, reflecting the complexity of SA/DP. The Swedish social security system fosters this flexibility by distinguishing between different situations of temporarily and permanently reduced work capacity and allowing for flexible work arrangements to remain in paid work with part-time SA/DP.

We observed that PwMS not only progressed from activity to full-time SA/DP, but some sequences within Stable High Activity and Unstable Medium Activity types had SA/DP around MS diagnosis with subsequent return to activity. Importantly, individuals with the Mixed: SA/ DP 30-180 days state were roughly equally as likely to remain in this state, progress to Mixed: SA/DP 180+ days, but also to return to Activity. This finding highlights that for some PwMS, return to work processes are relevant and reductions in work capacity can be temporary. These transitions back to activity were observed mostly after SA/ DP proximate to MS diagnosis. Similarly, around 5\% of a cohort of PwMS were previously observed to have a trajectory of decreasing SA/DP in the years after MS diagnosis following a peak at diagnosis. ${ }^{32}$

Membership characteristics differed across the types of working-life sequences. The trends of being a woman, lower education level and of older ages, being associated with types characterised by a higher extent of SA/DP reflect wider trends of SA/DP in Sweden ${ }^{33}$ and previous MS-specific findings. ${ }^{32}$ Unstable Medium Activity had characteristics more alike Stable High Activity than types characterised by more SA/DP. Consistent with our findings of membership to a type of sequence, women with MS have been observed to have more SA/DP than men with MS, ${ }^{32}{ }^{35}$ reflecting wider trends in Sweden. ${ }^{33} 34$ However, being a man is associated with a worse clinical course $^{36}$ emphasising the importance of both work situation and morbidity in assessing work capacity.

The observed associations of lower educational levels and types of sequences characterised by SA/DP follow previous SA/DP findings among PwMS. ${ }^{378}$ Likewise were the findings of higher odds for those working in manual labour positions to have a different type of working-life sequence than Stable High Activity compared with office workers. ${ }^{38}$ The combination of which suggests that the ability of individuals to adapt to MS symptoms and maintain work capacity early in the clinical course may differ at least in part by modifiable characteristics like educational level and type of occupation. This could be due to the work situations of more physically demanding tasks and/ or less flexible working arrangements that these characteristics may reflect, which may be unsustainable with MS progression. ${ }^{32}$

Anxiety and depression are common diagnoses among PwMS. ${ }^{139}$ A 3.6 higher odds of being prescribed antidepressants has been observed among PwMS in Sweden compared with non-MS individuals, with $25 \%$ of PwMS prescribed antidepressants. ${ }^{40}$ In our cohort, 23.9\% were prescribed medications for anxiety/depression in the MS diagnosis year, however, with slightly different definitions. In agreement with previous findings,${ }^{40}$ members of types of working-life sequences involving SA/DP had higher odds of anxiety/depression medications. Our findings emphasise the importance of anxiety/depression as a factor underlying the heterogeneity in PwMS' working life.

Further, individuals with Stable High SA/DP sequences were less likely than those with Stable High Activity sequences to have MS drugs in the MS diagnosis year. This was somewhat attenuated when adjusting for age (in addition to sex and MS diagnosis year) which is strongly associated with both MS disability milestones ${ }^{36}$ and DP. ${ }^{33}$ The most likely explanation is that more of these individuals had progressive MS types or alternatively had infusionbased MS drugs which were not included in the SPDR. The observed heterogeneity in working-life sequences may also reflect differences in MS disability, both between PwMS and at different time points for an individual. ${ }^{41}$ Further studies are required to disentangle the associations of MS progression and age on PwMS' working life.

Consistent with previous studies, ${ }^{3}{ }^{42} 43$ we found that overall PwMS' economic welfare did not decline in the five years following MS diagnosis. Nonetheless, the different state transitions observed largely represented decreasing work capacity and increasing sequence instability. There is a potential limit to this observed responsiveness of the SA/DP benefits to reductions in income from paid work, as we found non-significant differences in DI among the Stable High SA/DP type of sequences. With longer follow-up and further disease progression, reductions in annual DI are possible due to SA/DP payments being a proportion of previous income, and lower rates of compensation for DP than SA. ${ }^{343}$

Strengths of this study include the longitudinal design and use of high-quality nationwide registers to identify the PwMS and classify their working-life sequences without self-reports affected by recall bias. ${ }^{13} 16$ Through sequence analysis, as much individual-level information as possible was retained to describe working life, a complex phenomenon accounting simultaneously for multiple outcomes. The population-based design minimised selection bias by including all working-aged PwMS. Generalisability may be limited in countries with differently operating social security systems or labour markets. PwMS in Sweden has self-reported being in work to a higher extent than PwMS in other European countries. ${ }^{37}$ Sweden has in general high levels of employment among both women and men, including at older ages, ${ }^{44}$ limiting healthy worker selection in our cohort already at inclusion.

Sequence analysis is an exploratory method, ${ }^{9}$ providing important descriptive insights in sequences and elucidating recurrent patterns, ${ }^{30}$ with adjustment possible in subsequent regression analyses. Two assumptions 
were made when assigning a sequence to the relevant type of working-life sequence (ie, cluster solution) for the regression analyses: first, the differences between the sequences within each type were ignored; and the types of working-life sequences were treated as equally different from one another. ${ }^{30}$ There was a possible risk of misclassification when categorising the individuals, both in assigning the annual states and the type of sequence. Furthermore, choices in specification were necessary to conduct sequence analysis, including the states; dissimilarity measure and costs; and clustering method. Optimal matching was selected for the dissimilarity measure as it differentiated the timing of states in the sequence (ie, in relation to MS diagnosis). ${ }^{45}$ Transition matrices have been previously recommended when analysing career sequences. ${ }^{2629}$ Currently, there are no guidelines on the appropriate sample size, minimum sequence length or optimal number of states. ${ }^{28}$ We considered different cluster solutions and sensitivity analyses were conducted including an additional state.

Furthermore, population-based MS-specific information on MS disability, treatments, disease type and exact date of diagnosis was not available in our data. Our conservative definition of MS diagnosis from first ever MS ICD code strengthened assumptions regarding recentness and accuracy of the diagnosis. Nonetheless, some individuals may have been misclassified as first diagnosed with MS during the inclusion years despite already having the diagnosis, which could lead to an overestimation of activity states associated with further MS progression. The length of the study period was reduced to include a recent MS cohort with current treatments and social security regulations.

\section{CONCLUSION}

Sequence analysis assessed PwMS' working life by describing the patterns of activity and SA/DP in the first half-decade of MS course through identification of six types of working-life sequences. There was increasing diversity and instability with time from MS diagnosis in the sequences among those who transitioned into states involving SA/DP, reflecting temporal changes in work capacity among PwMS. The type of working-life sequences with largest membership had sequences predominantly of activity. The other types of working-life sequences identified had SA/DP to different extents and timing within the sequences and summarise different groups at risk for reducing activity early in the clinical course. These findings may inform interventions to maintain activity among PwMS. In addition, we conclude that sequence analysis is an efficient approach to identify factors associated with working life among PwMS.

Contributors $\mathrm{CM}$ : conceptualisation, methodology, formal analysis, writing-original draft. PT and KK: conceptualisation, methodology, writing-review and editing. $\mathrm{KA}$ : conceptualisation, methodology, resources, data curation, writing-review and editing, project administration, funding acquisition. JH: conceptualisation, methodology, writing-review and editing, funding acquisition. EF: conceptualisation, methodology, writing-review and editing, supervision.

Funding This work was supported by unrestricted grants from Biogen (number: 4-2803/2019) and the Swedish Research Council for Health, Working Life and Welfare (grant number: 2007-1762). The design of the study, data collection, analyses, interpretations of data, and writing of manuscript were performed without involvement of the funding bodies. Biogen was given the opportunity to comment on the manuscript before submission.

Competing interests $\mathrm{CM}, \mathrm{KA}$, and EF were partly funded by Biogen. KA has received unrestricted researcher-initiated grants from Biogen. EF has received unrestricted researcher-initiated grants from Celgene. PT has previously received salaries partly funded by Biogen. KK is only affiliated with Karolinska Institutet, not receiving financial compensation for her involvement in this study; she is working full time at Gilead Sciences AB. JH received honoraria for serving on advisory boards for Biogen and Novartis and speaker's fees from Biogen, Merck-Serono, Bayer-Schering, Teva and Sanofi-Aventis. He has served as P.I. for projects sponsored by, or received unrestricted research support from, Biogen, MerckSerono, TEVA, Novartis, and Bayer-Schering. His MS research is also funded by the Swedish Research Council.

Patient consent for publication Not required.

Ethics approval Due to the use of pseudonymised data from total population registers, informed consent from the research participants was not applicable. The project received ethical approval from the Regional Ethical Review Board in Stockholm. Approval numbers: 2007/762-31; 2009/23-32; 2009/1917-32; 2010/466-32; 2011/806-32; 2011/1710-32; and 2014/236-32.

Provenance and peer review Not commissioned; externally peer reviewed.

Data availability statement No data are available. Please contact Professor Kristina Alexanderson (kristina.alexanderson@ki.se) about why the data, according to the General Data Protection Regulation, the Swedish Data Protection Act, the Swedish Ethical Review Act, and the Swedish Public Access to Information and Secrecy Act, cannot be made available.

Supplemental material This content has been supplied by the author(s). It has not been vetted by BMJ Publishing Group Limited (BMJ) and may not have been peer-reviewed. Any opinions or recommendations discussed are solely those of the author(s) and are not endorsed by BMJ. BMJ disclaims all liability and responsibility arising from any reliance placed on the content. Where the content includes any translated material, BMJ does not warrant the accuracy and reliability of the translations (including but not limited to local regulations, clinical guidelines, terminology, drug names and drug dosages), and is not responsible for any error and/or omissions arising from translation and adaptation or otherwise.

Open access This is an open access article distributed in accordance with the Creative Commons Attribution Non Commercial (CC BY-NC 4.0) license, which permits others to distribute, remix, adapt, build upon this work non-commercially, and license their derivative works on different terms, provided the original work is properly cited, appropriate credit is given, any changes made indicated, and the use is non-commercial. See: http://creativecommons.org/licenses/by-nc/4.0/.

ORCID iD

Chantelle Murley http://orcid.org/0000-0003-4150-4275

\section{REFERENCES}

1 Brundin L, Kobelt G, Berg J, et al. New insights into the burden and costs of multiple sclerosis in Europe: results for Sweden. Mult Scler 2017;23:179-91.

2 Giovannoni G, Butzkueven H, Dhib-Jalbut S, et al. Brain health: time matters in multiple sclerosis. Mult Scler Relat Disord 2016;9 Suppl 1:S5-48.

3 Pfleger CCH, Flachs EM, Koch-Henriksen N. Social consequences of multiple sclerosis (1): early pension and temporary unemployment-a historical prospective cohort study. Mult Scler 2010;16:121-6.

4 Gyllensten H, Wiberg M, Alexanderson K, et al. How does work disability of patients with MS develop before and after diagnosis? A nationwide cohort study with a reference group. BMJ Open 2016;6:e012731.

5 Wiberg M, Friberg E, Stenbeck M, et al. Sources and level of income among individuals with multiple sclerosis compared to the general population: a nationwide population-based study. Mult Scler 2015;21:1730-41. 
6 Landfeldt E, Castelo-Branco A, Svedbom A, et al. The long-term impact of early treatment of multiple sclerosis on the risk of disability pension. J Neurol 2018;265:701-7.

7 Alexanderson K, Norlund A. Swedish Council on technology assessment in health care (SBU). Chapter 1. Aim, background, key concepts, regulations, and current statistics. Scand J Public Health Suppl 2004;63:12-30.

8 Fattore G, Lang M, Pugliatti M. The Treatment experience, burden, and unmet needs (TRIBUNE) study - measuring the socioeconomic consequences of multiple sclerosis. Mult Scler 2012;18:5-6.

9 Lindholdt L, Labriola M, Nielsen CV, et al. Sequence analysis to assess labour market participation following vocational rehabilitation: an observational study among patients sick-listed with low back pain from a randomised clinical trial in Denmark. BMJ Open 2017;7:e015661.

10 Perhoniemi R, Blomgren J, Laaksonen M. Sources of income following a rejected disability pension application: a sequence analysis study. Disabil Rehabil 2020;42:2161-9.

11 McLeod CB, Reiff E, Maas E, et al. Identifying return-to-work trajectories using sequence analysis in a cohort of workers with work-related musculoskeletal disorders. Scand J Work Environ Health 2018;44:147-55.

12 Abbott A. Sequence analysis: new methods for old ideas. Annu Rev Sociol 1995;21:93-113.

13 Ludvigsson JF, Andersson E, Ekbom A, et al. External review and validation of the Swedish national inpatient register. BMC Public Health 2011;11:450

14 Brooke HL, Talbäck M, Hörnblad J, et al. The Swedish cause of death register. Eur J Epidemiol 2017;32:765-73.

15 Wettermark B, Hammar N, Fored CM, et al. The new Swedish prescribed drug register--opportunities for pharmacoepidemiological research and experience from the first six months. Pharmacoepidemiol Drug Saf 2007;16:726-35.

16 Ludvigsson JF, Svedberg P, Olén O, et al. The longitudinal integrated database for health insurance and labour market studies (LISA) and its use in medical research. Eur J Epidemiol 2019;34:423-37.

17 Hillert J, Stawiarz L. The Swedish MS registry - clinical support tool and scientific resource. Acta Neurol Scand 2015;132:11-19.

18 Alping P, Piehl F, Langer-Gould A, et al. Validation of the Swedish multiple sclerosis register: further improving a resource for Pharmacoepidemiologic evaluations. Epidemiology 2019;30:230-3.

19 Statistika centralbyrån. Price basic amount: Statistics Sweden, 2019. Available: https://www.scb.se/en/finding-statistics/statisticsby-subject-area/prices-and-consumption/consumer-price-index/ consumer-price-index-cpi/pong/tables-and-graphs/price-basicamount/price-basic-amount/

20 Statistika centralbyrån. Harmonized index of consumer prices (2015=100): Statistics Sweden, 2019. Available: https://www.scb. se/en/finding-statistics/statistics-by-subject-area/prices-andconsumption/consumer-price-index/consumer-price-index-cpi/pong/ tables-and-graphs/harmonized-index-of-consumer-prices-hicp/hicp2015100/

21 Eurostat. Euro/ECU exchange rates - annual data, 2018. Available: https://appsso.eurostat.ec.europa.eu/nui/show.do?dataset=ert_bil_ eur_a\&lang=en

22 Statistika centralbyrån. Longitudinal integration database for sick leave and labour market studies (LISA) 1990-2013. (Longitudinell integrationsdatabas för sjukförsäkrings- och arbetsmarknadsstudier (LISA) 1990-2013 in Swedish). Örebro, Sweden: Statistics Sweden, 2016.

23 Lalic S, Bell JS, Gyllensten H, et al. Trajectories of sickness absence and disability pension before and after opioid initiation for noncance pain: a 10-year population-based study. Pain 2019;160:1224-33.

24 Pratt NL, Kerr M, Barratt JD, et al. The validity of the Rx-Risk comorbidity index using medicines mapped to the anatomical therapeutic chemical (ATC) classification system. BMJ Open 2018;8:e021122.
25 Brzinsky-Fay C. Lost in transition? labour market entry sequences of school Leavers in Europe. Eur Sociol Rev 2007;23:409-22.

26 Studer M, Ritschard G. A comparative review of sequence dissimilarity measures. LIVES Working Papers 2014;33https:// archive-ouverte.unige.ch/unige:78575 doi:10.12682/ lives.2296-1658.2014.33

27 Gabadinho A, Ritschard G, Müller NS, et al. Analyzing and Visualizing State Sequences in $R$ with TraMineR. J Stat Softw 2011;40:37.

28 Biemann T, Datta DK. Analyzing sequence data: optimal matching in management research. Organizational Research Methods 2014;17:51-76.

29 Dlouhy K, Biemann T. Optimal matching analysis in career research: a review and some best-practice recommendations. J Vocat Behav 2015;90:163-73.

30 Studer M. Weightedcluster library manual: a practical guide to creating typologies of trajectories in the social sciences with R. LIVES Working Papers 2013;24 https://www.lives-nccr.ch/en/ publication/weightedcluster-library-manual-practical-guide-creatingtypologies-trajectories-social doi:10.12682/lives.2296-1658.2013.24

31 Gottberg K, Einarsson U, Ytterberg C, et al. Health-Related quality of life in a population-based sample of people with multiple sclerosis in Stockholm County. Mult Scler 2006;12:605-12.

32 Björkenstam C, Alexanderson K, Wiberg M, et al. Heterogeneity of sickness absence and disability pension trajectories among individuals with MS. Mult Scler J Exp Transl Clin 2015;1:205521731559563.

33 Försäkringskassan. Social insurance in figures 2015, 2015. Swedish social insurance agency. Available: https://www.forsakringskassan. se/wps/wcm/connect/e37b4ff1-d5d2-42dc-896b-bac5c59390e6/ socialforsakringen_i_siffror_2015_engelsk.pdf?MOD=AJPERES

34 Samuelsson Å, Alexanderson K, Ropponen A, et al. Incidence of disability pension and associations with socio-demographic factors in a Swedish twin cohort. Soc Psychiatry Psychiatr Epidemiol 2012;47:1999-2009.

35 Tinghög P, Hillert J, Kjeldgård L, et al. High prevalence of sickness absence and disability pension among multiple sclerosis patients: a nationwide population-based study. Mult Scler 2013;19:1923-30.

36 Manouchehrinia A, Beiki O, Hillert J. Clinical course of multiple sclerosis: a nationwide cohort study. Mult Scler 2017;23:1488-95.

37 Kobelt G, Langdon D, Jönsson L. The effect of self-assessed fatigue and subjective cognitive impairment on work capacity: the case of multiple sclerosis. Mult Scler 2019;25:740-9.

38 Wiberg M, Murley C, Tinghög P, et al. Earnings among people with multiple sclerosis compared to references, in total and by educational level and type of occupation: a population-based cohort study at different points in time. BMJ Open 2019;9:e024836.

39 Marrie RA, Reingold S, Cohen J, et al. The incidence and prevalence of psychiatric disorders in multiple sclerosis: a systematic review. Mult Scler 2015;21:305-17.

40 Brenner P, Alexanderson K, Björkenstam C, et al. Psychiatric diagnoses, medication and risk for disability pension in multiple sclerosis patients; a population-based register study. PLoS One 2014;9:e104165

41 Confavreux C, Vukusic S. The clinical course of multiple sclerosis. Handb Clin Neurol 2014:122:343-69.

42 Murley C, Mogard O, Wiberg M, et al. Trajectories of disposable income among people of working ages diagnosed with multiple sclerosis: a nationwide register-based cohort study in Sweden 7 years before to 4 years after diagnosis with a population-based reference group. BMJ Open 2018;8:e020392.

43 Landfeldt E, Castelo-Branco A, Svedbom A, et al. Personal income before and after diagnosis of multiple sclerosis. Value Health 2018;21:590-5.

44 Statistiska centralbyrån. Women and men in Sweden 2018. Örebro, Sweden: Statistics Sweden, 2018.

45 Studer M, Ritschard G. What matters in differences between life trajectories: a comparative review of sequence dissimilarity measures. J R Stat Soc Ser A Stat Soc 2016;179:481-511. 